\title{
Fibre Bundles and Supersymmetries
}

\author{
R. G. Yates \\ Theoretical Physics Group Imperial College of Science and Technology, London SW7 2BZ, UK
}

\begin{abstract}
By extending the frame bundle of a manifold admitting a spin structure to a principal fibre bundle with group the super-Poincaré group we are able to derive the supergravity Lagrangian and its invariances under supersymmetry and other transformations in a global form.
\end{abstract}

\section{Introduction}

Since geometric formulations have proved so successful in modern physics it was natural that once the concept of supersymmetries had been accepted it would be attempted to recast the component formulations [1] in terms of a more geometric language. So far the closest way to achieve this aim has been the idea of superspace [2]: the extension of space-time to include four extra fermionic dimensions together with the differential geometry implied by the increased structure.

However, this approach suffers from various defects. First it seems that to agree with the results derived by more direct methods it is necessary to abandon the obvious generalization of the Levi-Civita connection and impose an ad hoc torsion condition [3]. Second, the theory includes a large number of extraneous fields whose interpretation poses difficulties. Third, no great simplification in terms of concepts required or algebra needing to be done is achieved. Lastly, the transformation used in superspace mix up the bosonic and fermionic variables leading to difficulties in interpretation and in identification of the theory with the seemingly bosonic space-time we actually inhabit.

In this paper we present a formulation of super-gravity in terms of fibre bundles [4] which we claim overcomes the objections to the concept of superspace. By considering a fibre bundle whose base space is space-time no difficulty with nilpotent co-ordinates arises, the transformations corresponding to those in superspace always acting in another space. The only fields arising are the vierbein $e_{\mu}^{a}$ and the spin $3 / 2$ field $\psi_{\mu}^{a}$, with the connection $\omega_{\mu}^{a b}$ being given by an obvious generalisation of the normal Levi-Civita $\omega_{\mu}^{a b}$. The supergravity Lagrangian and its invariance under local supersymmetry transformations can be quite easily derived. 
As the theory is best expressed in terms of fibre bundles and differential forms we use this language throughout [5] though expressions in terms of a local coordinate system are given at the end for those physicists not yet familiar with this notation.

\section{The Formalism and the Lagrangian}

As is well known over any four dimensional manifold $M$ there may exist many principal fibre bundles with group the proper Lorentz group $H$. In what follows we may choose any one of these though naturally for physics we are interested in one constructed from the frame bundle of $M$ via some metric, that is in a bundle of orthonormal frames with respect to that metric. Using such a bundle we wish to construct a principal fibre bundle whose group is the extended Poincare group, which we represent as the following set of $9 \times 9$ matrices:

$$
\left(\begin{array}{ccc}
R(S)^{a b} & {\left[\frac{1}{2} \lambda C \gamma^{a} S\right]^{b}} & p^{a} \\
0 & S^{a b} & \lambda^{a} \\
0 & 0 & 1
\end{array}\right)
$$

where $S^{a b}$ is an $\operatorname{SL}(2, C)$ matrix, $R$ is the usual map from $\operatorname{SL}(2, C)$ to the Lorentz group, $p^{a}$ is a four vector (the translation vector), $\lambda^{a}$ is an anticommuting spinor and $C$ is the charge conjugation matrix $\left(C \gamma^{a}=-\gamma^{a^{T}} C, C^{T}=-C\right)$. Letting the matrix $(2.1)$ be represented by $\left(S, p^{a}, \lambda\right)$ it is easily seen that they form a group with multiplication

$$
\left(S, p^{a}, \lambda\right)\left(U, q^{a}, \theta\right)=\left(\mathrm{SU}, p^{a}+(R(S) q)^{a}+\frac{1}{2} \lambda C \gamma^{a} S \theta, S \theta+\lambda\right) .
$$

Further they can be thought of as being generated by the super-Poincaré algebra.

$$
\begin{aligned}
{\left[J_{a b}, J_{c d}\right] } & =\eta_{b c} J_{a d}+\eta_{a d} J_{b c}-\eta_{a c} J_{b d}-\eta_{b d} J_{a c} \\
{\left[J_{a b}, P_{c}\right] } & =\eta_{b c} P_{a}-\eta_{a c} P_{b} \\
\left\{Q_{a}, Q_{b}\right\} & =\left(C \gamma^{c}\right)_{a b} P_{c} \\
{\left[J_{a b}, Q_{c}\right] } & =\left(\sigma_{a b}\right)^{d} Q_{c} \quad \sigma_{a b}=\frac{1}{4}\left[\gamma_{a}, \gamma_{b}\right],
\end{aligned}
$$

where

$$
\begin{aligned}
J_{a b} & =\left(\begin{array}{ccc}
j_{a b} & 0 & 0 \\
0 & \sigma_{a b} & 0 \\
0 & 0 & 0
\end{array}\right), \\
P_{a} & =\left(\begin{array}{ccc}
0 & 0 & P_{a} \\
0 & 0 & 0 \\
0 & 0 & 0
\end{array}\right) \\
Q_{a} & =\left(\begin{array}{ccc}
0 & \left(q_{a} C \gamma^{i}\right)_{j} & 0 \\
0 & 0 & q_{a} \\
0 & 0 & 0
\end{array}\right),
\end{aligned}
$$


with $\eta_{b c}=\operatorname{diag}(-1,1,1,1)$ and $j_{a b}$ the generators of the Lorentz group (before exponentiating $Q_{a}$ must be multiplied by an anticommuting parameter).

To extend the Lorentz bundle we use the construction technique given in Steenrod [6]. A fibre bundle can be constructed once its transition functions are known that is if we are given a covering of the base manifold $\left\{U_{i}\right\}$ say and a set of functions $f_{i j}: U_{i} \cap U_{j} \rightarrow G^{\prime}, G^{\prime}$ the group of interest, satisfying

$$
f_{i j}(x) f_{j k}(x)=f_{i k}(x) \text {. }
$$

If $G^{\prime}$ is a subgroup of $G$ then the bundle can be extended to one with group $G$ simply by regarding the transition functions as taking values in $G$ rather than $G^{\prime}$. For our case the situation is slightly more complicated due to the fact to view the Lorentz group as a subgroup of the super-Poincaré we must construct an inverse to $R$, but as this is a 2 to 1 mapping this may not be possible globally. However, we may certainly do this if $M$, the base space, admits a spin structure [7], so from now on we assume this is so. It is possible that the use of generalized spin structures [8] may allow us to proceed further if the second Stieffel-Whitney class of $M$ does not vanish. Having extended the $f_{i j}$ to take values in the super-Poincaré group we construct a new bundle $(E, \pi, M)$ with that as its group.

Having constructed our bundle we endow it with a connection, i.e. a one form taking values in the super-Poincaré algebra (2.4).

$$
\Gamma=\frac{1}{2} \omega^{a b} J_{a b}+\theta^{a} P_{a}+\psi^{a} Q_{a} .
$$

Where we have expressed the connection as a sum of those generators of the algebra which carry an immediate interpretation: $J_{a b}$ generate the $\operatorname{SL}(2, C)$ subgroup, $P_{a}$ are the bosonic translations and $Q_{c}$ the fermionic ones. As noted after Eq. (2.4) for $\Gamma$ to lie in the algebra $\psi^{a}$ must be an anticommuting spinor form and from the multiplication law (2.2) $\theta^{a}$ is an even element of a Grassman algebra. If $\sigma$ is a section from subset $U$ of $M$ to $E$ we may pull-back (2.6) to obtain

$$
\begin{aligned}
\sigma^{*} \omega^{a b} & =\omega_{\mu}^{a b} d x^{\mu}, \\
\sigma^{*} \theta^{a} & =e_{\mu}^{a} d x^{\mu}, \\
\sigma^{*} \psi^{a} & =\psi_{\mu}^{a} d x^{\mu},
\end{aligned}
$$

and we will later identify $\omega_{\mu}^{a b}$ as the Levi-Civita connection, $e_{\mu}^{a}$ as the vierbein and $\psi_{\mu}^{a}$ as the spin $3 / 2$ field normally used in the construction of supergravity. However, it should be noted that $\psi_{\mu}^{a}$ is not what one would normally think of as a spinor field. Usually this is a cross-section of some spin bundle whereas our $\psi$ field is a connection form.

The curvature two-form $\Delta$ is, as usual, given by the structure equation

$$
\Delta=d \Gamma+\frac{1}{2}[\Gamma, \Gamma]
$$

whence writing

$$
\Delta=\frac{1}{2} \Omega^{a b} J_{a b}+T^{a} P_{a}+\mathscr{T}^{a} Q_{a}
$$


we find

$$
\begin{aligned}
\Omega^{a b} & =d \omega^{a b}+\omega^{a c} \wedge \omega_{c}^{b}, \\
T^{a} & =d \theta^{a}+\omega^{a c} \wedge \theta_{c}+\frac{1}{2} \psi \wedge C \gamma^{a} \psi, \\
\mathscr{T}^{a} & =d \psi^{a}+\frac{1}{2} \omega^{b c} \wedge^{a}\left(\sigma_{b c} \psi\right) .
\end{aligned}
$$

Latin indices are raised and lowered using $\eta_{a b}$. Finally, from the Bianchi identity

$$
D \Delta=0 \text {, }
$$

where $D$ is the horizontal exterior derivative we deduce

$$
\begin{gathered}
d \Omega^{a b}+\omega^{a c} \wedge \Omega^{c b}-\Omega^{a c} \wedge \omega^{c b}=0, \\
d T^{a}+\omega^{a c} \wedge T^{c}-\Omega^{a c} \wedge \theta^{c}+\psi \wedge C \gamma^{a} \mathscr{T}=0, \\
d \mathscr{T}^{a}+\frac{1}{2} \omega^{b c} \wedge{ }^{a}\left(\sigma_{b c} \mathscr{T}\right)-\frac{1}{2} \Omega^{b c} \wedge{ }^{a}\left(\sigma_{b c} \psi\right) .
\end{gathered}
$$

To rewrite these in a more familiar notation we use the section $\sigma$ previously mentioned. Letting

$$
\begin{aligned}
\sigma^{*} \Omega^{a b} & =\frac{1}{2} R_{\mu \nu}^{a b} d x^{\mu} \wedge d x^{\nu}, \\
\sigma^{*} T^{a} & =\frac{1}{2} T_{\mu \nu}^{a} d x^{\mu} \wedge d x^{\nu}, \\
\sigma^{*} \mathscr{T}^{a} & =\frac{1}{2} \mathscr{T}_{\mu \nu}^{a} d x^{\mu} \wedge d x^{\nu},
\end{aligned}
$$

it can be seen that $(2.10)$ and (2.12) become $\underset{\mu, v, \alpha}{\mathscr{C}}$ means a cyclic sum over $\mu, v$, and $\alpha)$

$$
\begin{aligned}
& R_{\mu \nu}^{a b}=\partial_{\mu} \omega_{\nu}^{a b}-\partial_{\nu} \omega_{\mu}^{a b}+\omega_{\mu}^{a c} \omega_{v}^{c b}-\omega_{v}^{a c} \omega_{\mu}^{c b}, \\
& T_{\mu \nu}^{a}=\partial_{\mu} e_{\nu}^{a}-\partial_{v} e_{\mu}^{a}+\omega_{\mu c}^{a} e_{v}^{c}-\omega_{\mu c}^{a} e_{\mu}^{c}+\psi_{\mu} C \gamma^{a} \psi_{\nu}, \\
& \mathscr{T}_{\mu \nu}^{a}=\partial_{\mu} \psi_{\nu}^{a}-\partial_{\nu} \psi_{\mu}^{a}+\frac{1}{2} \omega_{\mu}^{e f a}\left(\sigma_{e f} \psi_{\nu}\right)-\frac{1}{2} \omega_{\nu}^{e f} a\left(\sigma_{e f} \psi_{\mu}\right) \text {, } \\
& \stackrel{\mathscr{C}, \alpha, \alpha}{\mu}\left\{\partial_{\mu} R_{v \alpha}^{a b}+\omega_{\mu c}^{a} R_{v \alpha}^{c b}-\omega_{\mu c}^{b} R_{v \alpha}^{c a}\right\}=0, \\
& \underset{\mu, v, \alpha}{\mathscr{C}}\left\{\partial_{\mu} T_{v \alpha}^{a}+e_{\mu c} R_{v \alpha}^{c a}+\omega_{\mu c}^{a} T_{v \alpha}^{c}+\psi_{\mu} C \gamma^{a} \mathscr{T}_{v \alpha}\right\}=0, \\
& \underset{\mu, v, \alpha}{\mathscr{C}, \alpha}\left\{\partial \mathscr{T}_{\nu \alpha}^{a}-\frac{1}{2} a^{a}\left(\sigma_{c d} \psi_{\mu}\right) R_{v \alpha}^{c d}+\frac{1}{2} \omega_{\mu}^{c d} a_{c d}\left(\sigma_{c \alpha} \mathscr{T}_{v \alpha}\right)\right\}=0 .
\end{aligned}
$$

If the $4 \times 4$ matrix $e_{\mu}^{a}$ has an inverse, $e^{\mu a}$ say, so

$$
e_{\mu}^{a} e_{c}^{\mu}=\delta_{c}^{a} .
$$

(2.14ii) can be solved for $\omega_{\mu}^{a b}$ giving

$$
\begin{aligned}
\omega_{\mu}^{a b}= & \frac{1}{2}\left\{e^{\lambda b}\left(\partial_{\lambda} e_{\mu}^{a}-\partial_{\mu} e_{\lambda}^{a}\right)-e^{\lambda a}\left(\partial_{\lambda} e_{\mu}^{b}-\partial_{\mu} e_{\lambda}^{b}\right)\right. \\
& +e_{\mu}^{d} e^{\lambda a} e^{v b}\left(\partial_{\nu} e_{d \lambda}-\partial_{\lambda} e_{d \nu}\right)+e^{\lambda b} \psi_{\lambda} C \gamma^{a} \psi_{\mu} \\
& -e^{\lambda a} \psi_{\lambda} C \gamma^{b} \psi_{\mu}+e_{\mu}^{d} e^{\lambda a} e^{v b} \psi_{\nu} C \gamma^{d} \psi_{\lambda} \\
& \left.-e^{\lambda b} T_{\lambda \mu}^{a}+e^{\lambda a} T_{\lambda \mu}^{b}-e_{d \mu} e^{\lambda a} e^{v b} T_{v \lambda}^{d}\right\}
\end{aligned}
$$


A gauge transformation in this language is a different choice of section $\sigma^{\prime}$ from $U$ to $E$ which is related to the original $\sigma$ by

$$
\sigma^{\prime}(x)=\sigma(x) g(x),
$$

where $g$ is an element of the super-Poincaré group. With $g=(S, p, \lambda)$ and denoting pull-backs with respect to $\sigma^{\prime}$ by primes, it is found that $(R=R(S))$

$$
\begin{aligned}
\omega_{\mu}^{\prime}= & R^{-1} \omega_{\mu} R+R^{-1} \frac{\partial R}{\partial x^{\mu}}, \\
e_{\mu}^{a^{\prime}}= & \left(R^{-1} e_{\mu}+R^{-1} \frac{\partial p}{\partial x^{\mu}}+R^{-1} \omega_{\mu} p\right) \\
& +\psi_{\mu} C S \gamma^{a} S^{-1} \lambda-\frac{1}{2} \lambda C S \gamma^{a} S^{-1} \frac{\partial \lambda}{\partial x^{\mu}} \\
& -\frac{1}{4} \lambda C S \gamma^{a} S^{-1} \sigma_{c d} \lambda \omega_{\mu}^{c d}, \\
\psi_{\mu}^{\prime}= & S^{-1} \psi_{\mu}+S^{-1} \frac{\partial \lambda}{\partial x^{\mu}}+\frac{1}{2} S^{-1} \sigma_{c d} \lambda \omega_{\mu}^{c d}, \\
R_{\mu \nu}^{\prime}= & R^{-1} R_{\mu \nu} R, \\
T_{\mu \nu}^{a^{\prime}}= & \left(R^{-1} T_{\mu \nu}+R^{-1} R_{\mu \nu} p\right)+\mathscr{T}_{\mu \nu} C S \gamma^{a} S^{-1} \lambda \\
& -\frac{1}{4} R_{\mu \nu}^{c e} \lambda C S \gamma^{a} S^{-1} \sigma_{c e} \lambda, \\
\mathscr{T}_{\mu \nu}^{\prime}= & S^{-1} \mathscr{T}_{\mu \nu}+\frac{1}{2} R_{\mu \nu}^{c d} S^{-1} \sigma_{c d^{\prime}} .
\end{aligned}
$$

We now turn to the construction of a Lagrangian for the theory. For general relativity one can view the Einstein lagrangian as arising from the requirement that variations of it with respect to $\omega_{\mu}$ should lead to an equation stating that the torsion is zero. With $L$ being the lagrangian four-form the correct expression is

$$
\left.\delta L\right|_{\theta=\text { constant }}=2 \delta \omega^{c d} \wedge T^{a} \wedge \theta^{b} \varepsilon_{a b c d} .
$$

Using (2.10) and (2.12) with $\psi=\mathscr{T}=0$ this may be integrated to give

$$
L=\theta^{a} \wedge \theta^{b} \wedge \Omega^{c d} \varepsilon_{a b c d}
$$

whose pull-back yields a lagrangian density of

$$
\mathscr{L}=e R \text {. }
$$

$R$ being the scalar curvature and $e=\operatorname{det} e_{\mu}^{a}$. To obtain the supergravity lagrangian we need only to drop the condition that $\psi=0$ whence the extra term $\frac{1}{2} \psi \wedge C \gamma^{a} \psi$ in the torsion tensor $T^{a}$ leads to the expression

$$
L=\theta^{a} \wedge \theta^{b} \wedge \Omega^{c d} \varepsilon_{a b c d}-2 \psi \wedge C \gamma_{5} \gamma_{b} \sigma_{e f} \omega^{e f} \wedge \psi \wedge \theta^{b}
$$

The last term in (2.24) is not invariant under a gauge transformation induced by an element of SL $(2, C)$ but we may make it so by adding the term 
$-4 \psi \wedge C \gamma_{5} \gamma_{b} d \psi \wedge \theta^{b}$, which will not effect the variational Eq. (2.21), leading to a final expression

$$
L=\theta^{a} \wedge \theta^{b} \wedge \Omega^{c d} \varepsilon_{a b c d}-4 \psi \wedge C \gamma_{5} \gamma_{b} \mathscr{T} \wedge \theta^{b} .
$$

The pull-back of (2.25) then gives

$$
\mathscr{L}=e R+\psi_{\mu} C \gamma_{5} \gamma_{b} \mathscr{T}_{\nu \lambda} e_{\tau}^{b} \varepsilon^{\mu \nu \lambda \tau}
$$

which is the supergravity lagrangian [9].

To show the invariance of the lagrangian under supersymmetry transformations we will later need to know what $d L$ is. We find that

$$
d L=2 T^{a} \wedge\left(\theta^{b} \wedge \Omega^{c d} \varepsilon_{a b c d}+2 \psi \wedge C \gamma_{5} \gamma_{a} \mathscr{T}\right)
$$

This can be verified by direct computation using (2.10) and (2.12), but it can also be shown using more subtle ideas which will be of importance in deriving the supersymmetry transformations themselves.

Having constructed our total space $E$ we wish to consider the space $E / \mathrm{SL}(2, C)$; that is we identify those points in $E$ which are related by multiplication by an $\operatorname{SL}(2, C)$ matrix. Now $(E, p r, E / \operatorname{SL}(2, C))$, where pr is the obvious map from $E$ to $E / \operatorname{SL}(2, C)$ is a principal fibre bundle with group $\operatorname{SL}(2, C)$ and $\omega^{a b}$ is a connection form for it. In this bundle we denote the horizontal exterior derivative by $D$.

Equations (2.10) and (2.12) can now be written as

$$
\begin{aligned}
\Omega^{a b} & =D \omega^{a b}, \\
T^{a} & =D \theta^{a}+\frac{1}{2} \psi \wedge C \gamma^{a} \psi, \\
\mathscr{T} & =D \psi, \\
D \Omega & =0, \\
D T^{a} & =\Omega^{a c} \wedge \theta_{c}-\psi \wedge c \gamma^{a} \mathscr{T}, \\
D \mathscr{T} & =\frac{1}{2} \sigma_{b c} \Omega^{b c} \wedge \psi .
\end{aligned}
$$

Over this bundle $L$ is, by construction, an horizontal four form invariant by right multiplication of $\operatorname{SL}(2, C)$ and hence [5].

$$
d L=D L=2 T^{a} \wedge\left(\theta^{b} \wedge \Omega^{c d} \varepsilon_{a b c d}+2 \psi \wedge C \gamma_{5} \gamma_{a} \mathscr{T}\right)
$$

\section{Invariances of the Lagrangian}

To construct the supersymmetry transformations it is necessary to reduce the bundle $(E, \pi, M)$ to one whose group is $\operatorname{SL}(2, C)$. Although we started with a bundle whose group was the Lorentz group and, by the assumption of the existence of a spin structure, this could be extended to an $\operatorname{SL}(2, C)$ bundle, the process is not circular as the final objects we consider inherit the richer structure introduced in the construction of $E$. E/SL $(2, C)$ can be thought of as an associated bundle to $E$ over $M$ and by standard theorems the reduction of $E$ to $\operatorname{SL}(2, C)$ bundles are in one-one correspondence to cross-sections of this associated bundle. 
If $\sigma$ is a cross section of $(E / \operatorname{SL}(2, C), j, M)$ the reduced bundle is $\sigma^{-1} E C M \times E$ where $\sigma^{-1} E=\{(x, u)$ s.t. $\sigma(x)=\operatorname{pr}(u)\}$ i.e. Fig. 1 is commutative.

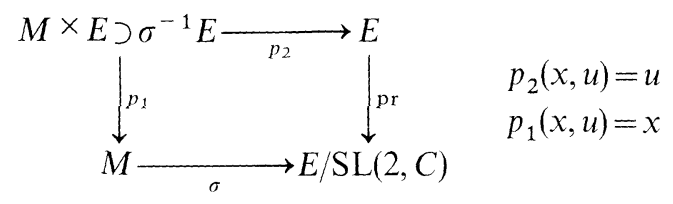

Fig. 1.

We require $\sigma$ to be such that the forms $\omega^{a b}$ and $\theta^{a}$ pulled back to $\sigma^{-1} E$ via $p_{2}$ are an absolute parallelism [essentially this means the matrix $\left(e_{\mu}^{a}\right)$ has an inverse]. This is a reasonable assumption as to make contact with physics we will need to identify $e_{\mu}^{a}$ as the vierbein which implies the existence of its inverse. More mathematically we have constructed a mapping from a Lorentz group bundle over $M$ to $E$ and the above requirement is that the pull-back of $\theta^{a}$ under this map is the canonical form of that bundle which, while being a restriction of the types of connections on $E$ we consider, is certainly possible.

As $\theta^{a}$ and $\omega^{a b}$ form an absolute parallelism by the standard constructions we can choose a new connection which is such that $T^{a}=0$ on $\sigma^{-1} E$ (we denote forms on $E$ and their pull-backs via $p_{2}: M \times E \rightarrow E$ by the same symbol) and we assume that this has been done. Now $M \times E$ has a natural group action by the superPoincaré group, namely $(x, u) \rightarrow(x, u g)$ and we consider the submanifolds $R_{g} \sigma^{-1} E$ defined by $(x, u) \in R_{g} \sigma^{-1} E$ if, and only if, $\sigma(x)=\operatorname{pr}\left(u g^{-1}\right)$. Clearly $R_{e} \sigma^{-1} E=\sigma^{-1} E$ and we claim that for each $g R_{g} \sigma^{-1} E$ is a principal fibre bundle over $M$ with group $\operatorname{SL}(2, C)$. This is nearly obvious: we need only state that the group action is

for clearly

$$
R_{h}(x, v)=\left(x, v g^{-1} h g\right) h \in \mathrm{SL}(2, C)
$$

$$
\sigma(x)=\operatorname{pr}\left(v g^{-1}\right)=\operatorname{pr}\left(v g^{-1} h\right)=\operatorname{pr}\left(v g^{-1} h g g^{-1}\right)
$$

so $\left(x, v g^{-1} h g\right)$ is in $R_{g} \sigma^{-1} E$ and the rest follows easily. Also obvious is the fact that we thus have a set of maps between equivalent $\operatorname{SL}(2, C)$ bundles over $M$.

Fig. 2

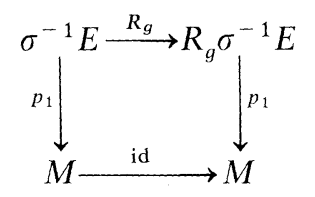

It is, perhaps, worth emphasizing that we cannot define an action of $G$ on the cross-section $\sigma$. Although each reduction of the bundle $E$ defines a cross-section of $(E, \mathrm{pr}, E / H)$ equivalent bundles define the same cross-section. The difference between $\sigma^{-1} E$ and $R_{g} \sigma^{-1} E$ lies in the different action of $H$ or better in the different representation of $H$ in $G$ used.

If $g \in \operatorname{SL}(2, C)$ clearly $R_{g} \sigma^{-1} E=\sigma^{-1} E$ and any change in the forms is just a gauge transformation which is not particularly interesting so we assume that the group element $g$ is either a bosonic or femionic translation i.e. generated by an element of the subalgebra spanned by the $P_{a}$ and $Q_{a}$ of (2.4). 
It is not necessarily true that the torsion form of $E, T^{a}$, pulled back to $R_{g} \sigma^{-1} E$ is zero, however, if $g$ is chosen so that $\omega^{a b}$ and $\theta^{a}$ on $R_{g} \sigma^{-1} E$ still form an absolute parallelism we can find a horizontal one form, $\phi^{a b}$ say, such that with respect to the connection $\omega^{\prime a b}=\omega^{a b}+\phi^{a b}$ the new torsion form is zero. The supersymmetry transformations are then the pull backs from $R_{g} \sigma^{-1} E$ of $\omega^{\prime a b}, \theta^{a}$, and $\psi^{a}$ by $R_{g}$ to $\sigma^{-1} E$.

To find the infinitesimal form of these transformations let $g=\exp t T$. As $g$ is not in the $\operatorname{SL}(2, C)$ subgroup we have by definition of $\omega$

$$
\omega(T)=0 .
$$

Let

$$
\begin{gathered}
\theta^{a}(T)=p^{a}, \\
\psi^{a}(T)=\lambda^{a} .
\end{gathered}
$$

Then the infinitesimal change in $\theta^{a}$ and $\psi^{a}$ will be given by Lie differentiation by $T$ and as $\phi^{a b}=0$ on $\sigma^{-1} E$ the first order change is $\omega$, namely

$$
\underset{t \rightarrow 0}{\mathscr{L}} \frac{1}{t}\left(\omega^{a b}-R_{\exp t T}^{*} \omega^{\prime a b}\right)=\underset{t \rightarrow 0}{\mathscr{L}} \frac{1}{t}\left(\omega^{\prime a b}-R_{\exp t T}^{*} \omega^{\prime a b}\right)=L_{T} \omega^{\prime a b},
$$

where $L_{T}$ denotes Lie differentiation. Using $L_{T}=d \circ l_{T}+l_{T} \circ d$ we find

$$
\begin{aligned}
\delta \theta^{a} & =L_{T} \theta^{a}=d \theta^{a}(T)+l_{T} d \theta^{a} \\
& =d p^{a}+\iota_{T}\left(T^{a}-\omega_{c}^{a} \wedge \theta^{c}-\frac{1}{2} \psi \wedge C \gamma^{a} \psi\right) \\
& =d p^{a}+\omega^{a}{ }_{c} p^{c}-\lambda C \gamma^{a} \psi, \\
\delta \psi^{a} & =L_{T} \psi^{a}=d \psi^{a}(T)+\imath_{T} d \psi^{a} \\
& =d \lambda^{a}+\imath_{T}\left(\mathscr{T}^{a}-\frac{1}{2} \omega^{b c a}\left(\sigma_{b c} \psi\right)\right) \\
& =d \lambda^{a}+\frac{1}{2} \omega^{b c a}\left(\sigma_{b c} \lambda\right),
\end{aligned}
$$

where we have used the fact that $T$ is a vertical vector of $E$ i.e. $\imath_{T}\left(T^{a}\right)=l_{T}\left(\mathscr{T}^{a}\right)=0$. Calculation of $\delta \omega^{a b}$ is more complicated as we need $L_{T} \omega^{\prime a b}$ which is not equal to $L_{T} \omega^{a b}$ which is of course zero. In a co-ordinate form $\delta \omega^{a b}$ may be calculated from the co-ordinate expression for the change in $e_{\mu}^{a}$ and $\psi_{\mu}^{a}$ to be derived below and Eq. (2.17) with $T_{\mu \nu}^{a}$ set equal to zero. For a co-ordinate-free expression we may use $L_{T} \omega^{\prime a b}=L_{T} \phi^{a b}\left(L_{T} \omega^{a b}=0\right)$ and the fact that $\phi^{a b}$ is given by

$$
\phi^{a b}=\sum_{k} \frac{1}{2} \theta^{k}\left(l_{X_{k}} l_{X_{b}} T^{a}+l_{X_{a}} l_{X_{k}} T^{b}+l_{X_{a}} l_{X_{b}} T^{k}\right)
$$

( $T^{a}$ can only be set equal to zero after the Lie differentiation has been performed) where $X_{i}$ are a dual set of vectors to $\theta^{a}$, i.e.

$$
\theta^{a}\left(X_{i}\right)=\delta_{i}^{a}
$$

Repeated applications of the formula

$$
L_{T} l_{X}=l_{X} L_{T}+l_{[T, X]}
$$


and the use of the Bianchi identity (2.12ii) leads to

$$
\begin{aligned}
\delta \omega^{a b}= & \sum_{k} \frac{1}{2} \theta^{k}\left(l_{X_{k}} l_{X_{b}} \Omega^{a c} p_{c}+l_{X_{a}}{ }^{{ } X_{k}} \Omega^{b c} p_{c}+l_{X_{a}} l_{X_{b}} \Omega^{k c} p_{c}\right. \\
& \left.-l_{X_{k}} l_{X_{b}} \lambda C \gamma^{a} \mathscr{T}-l_{X_{a}} l_{X_{k}} \lambda C \gamma^{b} \mathscr{T}-l_{X_{a}} l_{X_{b}} \lambda C \gamma^{k} \mathscr{T}\right) .
\end{aligned}
$$

It should be noted that (3.8), unlike (3.3) and (3.4) uses the fact that $T^{a}=0$ on $\sigma^{-1} E$. Under a pull back to $M p^{a}$ and $\lambda$ become arbitrary functions, of even and odd Grassman type respectively, of the physical co-ordinates of $M, x^{\mu}$ say, giving

$$
\begin{aligned}
& \delta e_{\mu}^{a}=\frac{\partial p^{a}}{\partial x^{\mu}}+\omega_{\mu c}^{a} p^{c}-\lambda C \gamma^{a} \psi_{\mu}, \\
& \delta \psi^{a}=\frac{\partial \lambda^{a}}{\partial x^{\mu}}+\frac{1}{2} \omega_{\mu}^{b c a}\left(\sigma_{b c} \lambda\right),
\end{aligned}
$$

which are the usual expressions for the local supersymmetry transformations without auxiliary fields. Equation (3.8) then gives

$$
\begin{aligned}
\delta \omega_{\mu}^{a b}= & \sum_{k} \frac{1}{2} e_{\mu}^{k}\left(R_{\lambda \tau}^{a c} p_{c} e^{\lambda b} e^{\tau k}+R_{\lambda \tau}^{b c} e^{\lambda k} e^{\tau a} p_{c}+R_{\lambda \tau}^{k c} p_{c} e^{\lambda b} e^{\tau a}\right. \\
& \left.-\lambda C \gamma^{a} \mathscr{T}_{\lambda \tau} e^{\lambda b} e^{\tau k}-\lambda C \gamma^{b} \mathscr{T}_{\lambda \tau} e^{\lambda k} e^{\tau a}-\lambda C \gamma^{k} \mathscr{T}_{\lambda \tau} e^{\lambda b} e^{\tau a}\right) .
\end{aligned}
$$

Turning to the effect on the lagrangian of these transformations we need to know $L_{T} L^{\prime}$ where the prime means that it is constructed using $\omega^{\prime a b}$. We find

$$
\begin{aligned}
\delta L & =L_{T} L^{\prime}=d l_{T} L^{\prime}+l_{T} d L^{\prime}=d l_{T} L^{\prime}+l_{T} D^{\prime} L \\
& =d l_{T} L^{\prime}+l_{T}\left(2 T^{a}\left(\theta^{b} \wedge \Omega^{c d} \varepsilon_{a b c d}+2 \psi \wedge C \gamma_{5} \gamma_{a} \mathscr{T}\right)\right) \\
& =d l_{T} L^{\prime} \quad \text { as } \quad T^{a}=0 \text { on } \sigma^{-1} E,
\end{aligned}
$$

i.e. the change in the lagrangian is a total divergence, and hence, if surface integrals are ignored, the supersymmetry transformations are an invariance of the equations of motion [9].

Most use of the invariances (3.3) and (3.4) have focussed on the case $p_{c}=0$, the common assumption being that the variation due to a non-zero $p_{c}$ is a gauge transformation. That this is not so can be seen by comparing (2.19i), the gauge transformation of $\omega^{a b}$, with (3.11). Actually with $\lambda=0 \psi=0$, (3.9) and (3.11) give the first order forms of an invariance of Einstein's equations independent of supersymmetry considerations, the group now being extended from the Lorentz to the Poincaré groups, not the super-Poincaré groups.

In fact from their construction one can easily see that neither the $p^{c}=0$ nor the $\lambda=0$ transformations can be thought of as gauge transformations and it is therefore not surprising when techniques of quantization developed for gauge theories, such as Fadeev-Popov ghosts, do not give reasonable results in the context of super-gravity [10]. It has been thought that such problems arise because the group generated by (3.3) and (3.4) is an infinite dimensional one. Using auxiliary fields, which for matter-free gravity have no physical significance at the classical level, it has implicitly been thought that the group has been reduced to some finite dimensional one but this is not true. The closest algebra to achieve this aim is the Stony Brook algebra [see 11] but this is also infinite dimensional due to 
the occurrence of the fields $e_{\mu}^{a}$ and $\psi_{\mu}^{a}$ in the structure functions: a finite algebra results only when the structure functions reduce to constants.

The above invariances are constructed using vertical vectors in $T E$ and it is then an obvious question to ask if there exist other invariances of the Lagrangian generated by horizontal vectors. As these invariances are immediately applicable to other gauge theories we may, for the present let $(E, \pi, M)$ be a principal fibre bundle over $M$ with arbitrary group $G$. If $L$ is a lagrangian for some theory based on the bundle it is an horizontal four form of $T^{*} E$ invariant under the action of $G$ and therefore projecting uniquely to a four form, which we also call $L$, on $T^{*} M$. Effectively this means if we know $L$ or a transformation of $L$ at one point of a fibre of $E$ we know it at all points of that fibre.

Let $f_{t}$ be a one parameter family of diffeomorphisms of $M$ generated by a vector field $X$. Then, by standard theorems, there exists a one parameter family of diffeomorphisms of $E \tilde{f}_{t}$ generated by the vector field $\tilde{X}$ satisfying

$$
\pi \circ \tilde{f}_{t}=f_{t}
$$

$$
\tilde{X} \text { is the horizontal lift of } X \text {, }
$$

i.e. $\tilde{f}_{t}$ "covers" $f_{t}$ and the tangent vectors to it are horizontal so Fig. 3 is a commutative diagram.

Fig. 3

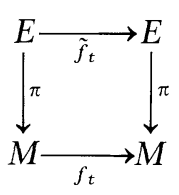

The invariance we are looking for is then

$$
L \rightarrow \tilde{f}_{t}^{*} L \text { in } E
$$

(note by construction $f_{t}^{*} L$ is a lagrangian form: that is it is horizontal and invariant by $R_{g}$ ), or equivalently

$$
L \rightarrow f_{t}^{*} L \quad \text { in } M .
$$

Clearly, since $f_{t}$ is a diffeomorphism

$$
\int_{M} f_{t}^{*} L=\int_{f_{t}(M)} L=\int_{M} L
$$

so the equations of motion are left unaltered by (3.14).

The interest of these transformations lies not so much in the co-ordinate free expression but in the local co-ordinate changes of the fields. Suppose we have used a section $s$ to pull-back the connection one form $\omega$ so

$$
s^{*} \omega=A_{\mu}^{a} d x^{\mu} T_{a},
$$

where $T_{a}$ are the Lie algebra matrices. To find the change in $A_{\mu}$ we need to calculate $s^{*} \tilde{f}_{t}^{*} \omega$. Now $s \circ f_{t}$ is again a section of the bundle but while $s\left[f_{t}(x)\right]$ and 
$\tilde{f}_{t}[s(x)]$ are in the same fibre, by the construction of $\tilde{f}_{t}$ they need not necessarily be the same point, that is Fig. 4 need not be a commutative diagram.

Fig. 4.

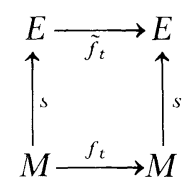

However, because $s\left[f_{t}(x)\right]$ and $\tilde{f}_{t}[s(x)]$ are in the same fibre there exists an element $g_{t}$ of $G$ such that

$$
s\left[f_{t}(x)\right] g_{t}(x)=\tilde{f}_{t}[s(x)]
$$

i.e.

$$
\tilde{f}_{t} \circ S=R_{g_{t}} s \circ f_{t}
$$

and therefore

$$
s^{*} \tilde{f}_{t}^{*} \omega=f_{t}^{*} s^{*}\left[\operatorname{ad} g_{t}^{-1} \omega\right]
$$

leading to

$$
A_{\mu}^{\prime}(x)=\left(g_{t}^{-1} A_{\alpha}\left(x^{\prime}\right) g_{t}+g_{t}^{-1} \frac{\partial g_{t}}{\partial x^{\prime \alpha}}\right) \frac{\partial x^{\prime \alpha}}{\partial x^{\mu}}
$$

where $x^{\prime}=f_{t}(x)$. To find $g_{t}$ we take the differential of (3.18) and use the Leibniz rule to obtain

$$
R_{g_{t}} s_{*} X+\left(s \circ f_{t}\right)_{*} \dot{g}_{t}=\tilde{X}
$$

where $\dot{g}_{t}$ is the tangent vector to the curve $u \rightarrow R_{g_{t}} u$. As we are working locally we may assume a local product structure to $E$ so the section $s$ can be written as

$$
s: x \rightarrow(x, h(x)) \quad h \in g
$$

and

$$
\omega_{j}^{i}=h_{k}^{-1 i}\left(d h_{j}^{k}+A_{\mu r}^{k} h_{j}^{r} d x^{\mu}\right)
$$

On applying $\omega$ to (3.21) we get a local differential equation for $g_{t}$ of the form

$$
\frac{d x^{\mu}}{d t}\left(g_{t}^{-1} h^{-1} A_{\mu} h g_{t}+g_{t}^{-1} h^{-1} \frac{\partial h}{\partial x^{\mu}} g_{t}+g_{t}^{-1} \frac{\partial g_{t}}{\partial x^{\mu}}\right)=0
$$

as

$$
\begin{aligned}
\omega\left(R_{g_{t}} S_{*} X\right) & =g_{t}^{-1} \omega\left(s_{*}\left(\frac{d x^{\mu}}{d t} \frac{\partial}{\partial x^{\mu}}\right)\right) g_{t} \\
& =g_{t}^{-1} \omega\left(\frac{d x^{\mu}}{d t} \frac{\partial}{\partial x^{\mu}}+\frac{d h_{j}^{i}}{d t} \frac{\partial}{\partial h_{j}^{i}}\right) g_{t}
\end{aligned}
$$


For physical applications it is implicitly assumed that the constant section $x \rightarrow(x, e), e$ the identity of $G$, has been chosen whence (3.24) becomes

which integrates to

$$
\frac{d x^{\mu}}{d t} A_{\mu} g_{t}+\frac{d x^{\mu}}{d t} \frac{\partial g_{t}}{\partial x^{\mu}}=0
$$

$$
g_{t}=P \exp \left(-\int_{c} A_{\mu} d x^{\mu}\right)
$$

where $P$ is the path ordering operator and $c$ is the curve from $x$ to $x^{\prime}$. Essentially (3.24) is important if we wished to globally patch together the change in $A_{\mu}$ or if $f_{t}$ were such that there did not exist a trivializing neighbourhood of the bundle containing $x$ and $x^{\prime}$, for then $s$ could not be defined and we would have to work from

$$
\omega^{\prime}=\tilde{f}_{t}^{*} \omega
$$

Such transformations as (3.20) have been considered by Jackiw [12] with $f_{t}$ being a conformal transformation of a flat space-time. In the language of this paper Jackiw was concerned with the difference between the invariances $f_{t}^{*} s^{*}$, the usual way of writing the conformal transformation of $A_{\mu}$, and $s^{*} \tilde{f}_{t}^{*}$ which he constructed by inspection (see Fig. 4). However, (3.28) gives a global definition of the invariance whereas, as pointed out above, the definition $f_{t}^{*} s^{*}$ can break down if $f_{t}$ is a "large" enough transformation to take us outside the domain of $s$.

If $L$ contains fields transforming under a representation $T$ of $G$, i.e. crosssections of associated bundles, they transform as

$$
\phi^{\prime}=T\left(g_{t}^{-1}\right) f_{t}^{*} \phi .
$$

To get the infinitesimal forms of these transformations let $X=f^{\alpha} \frac{\partial}{\partial x^{\alpha}}$ where $f^{\alpha}$ are arbitrary functions of $x^{\mu}$. Then to first order (3.27) becomes

$$
g_{t}=e-f^{\alpha} A_{\alpha}
$$

and (3.20) is seen to give

$$
\delta A_{\mu}=f^{\alpha} F_{\alpha \mu},
$$

where $F_{\alpha \mu}$ is the curvature form for $A_{\mu}$. (3.29) now becomes

$$
\delta \phi_{\alpha_{1} \ldots \alpha_{n}}=f^{\alpha} D_{\alpha} \phi_{\alpha_{1} \ldots \alpha_{n}}+\sum_{i} \phi_{\alpha_{1} \ldots \alpha_{i-1} \alpha_{i+1} \ldots \alpha_{n}} \frac{\partial f^{\alpha}}{\partial x^{\alpha_{i}}},
$$

where $D_{\alpha}$ is the appropriate gauge covariant derivative.

Specializing to the bundle $\left(\sigma^{-1} E, p_{1}, M\right)$ for supergravity this implies the following invariances of the Lagrangian $[G$ is now $\operatorname{SL}(2, C)]$.

$$
\begin{aligned}
\delta \omega_{\mu}^{a b} & =f^{\alpha} R_{\alpha \mu}^{a b}, \\
\delta e_{\mu}^{a} & =f^{\alpha} \frac{\partial e_{\mu}^{a}}{\partial x^{\alpha}}+f^{\alpha} \omega_{\alpha}^{a b} e_{b \mu}+e_{\alpha}^{a} \frac{\partial f^{\alpha}}{\partial x^{\mu}}, \\
\delta \psi_{\mu} & =f^{\alpha} \frac{\partial \psi_{\mu}}{\partial x^{\alpha}}+\frac{1}{2} f^{\alpha} \omega_{\alpha}^{a b} \sigma_{a b} \psi_{\mu}+\psi_{\alpha} \frac{\partial f^{\alpha}}{\partial x^{\mu}},
\end{aligned}
$$


where $f^{\alpha}$ can now be bosonic elements of a Grassmann algebra. For general relativity $(\psi=0)(3.33)$ is seen to be equivalent to (3.9) and (3.11), using the Bianchi identities with $T_{\mu \nu}^{a}=0$ and setting $f^{\alpha}=p^{c} e_{c}^{\alpha}$, but this is no longer true for supergravity as with $\lambda=0 \delta \psi=0$ by (3.10) but $\delta \psi=0$ for the transformation (3.33). The lagrangian remains invariant as the change in $\omega$ is different for the two transformations due to extra terms in $\mathscr{T}_{\mu v}$ in the Bianchi identities. Hence (3.33) are a new set of invariances for the supergravity lagrangian.

\section{Conclusions}

Using the notions of principal fibre bundles we have constructed the supergravity lagrangian and three invariances of it namely (3.9) and (3.10) with either $\lambda$ or $p_{c}$ equal to zero and (3.33). The former are special to the extended theory of gravity, depending on the existence of an absolute parallelism (i.e. the existence of an inverse for $\left.e_{\mu}^{a}\right)$, the resultant choice of the torsion tensor to be zero and the fact that the change in the lagrangian, up to a total divergence, is proportional to the torsion tensor. The transformations (3.33) are a special case of a set of invariances general to all gauge theories and, if the $\psi$ field is not identically zero, are not equivalent to the former. It should be noted that even if we start with $\psi=0(3.10)$ with a non-zero $\lambda$ gives us a non-zero spin $3 / 2$ field unlike (3.33). Most attention in the literature has focussed only on the invariances (3.9) and 10 with $p_{c}=0$ but if this is important for the theory and its quantization we can think of no reason why the transformations with $p_{c} \neq 0$ and the invariances (3.33) should not be of equal importance.

Using the fibre bundle construction technique we are able to avoid the conceptual difficulties of giving a meaning to the superspace translations that mix up bosonic and fermionic co-ordinates. At the same time various properties of the theory are made clear. First supersymmetry transformations are not gauge transformations. Secondly the $\psi$ field is not strictly speaking a spin $3 / 2$ field in the sense that it is part of a connection one form and should not be thought of as a cross-section to an associated bundle. This implies some interesting topological possibilities for the theory, to which we hope to return in a later publication.

Apart from global possibilities raised by the construction of the fibre bundle $E$ over $M$ it remains to see how matter fields, which would presumably be crosssections of some associated bundle to $E$, fit into this language as well as the problems of quantizing such theories. We feel, however, that the richness of structure and the fact that previously obtained results fit so naturally into the fibre bundle framework make this a promising line of approach to the problem of reconciling quantum theory with general relativity.

Acknowledgement. I would like to thank Chris Isham for helpful discussions and to acknowledge financial support from the Science Research Council of Great Britain under grant number NG 06586.

\section{References}

1. Wess, J., Zumino, B. : Nucl. Phys. B 70, 39-50 (1974)

Wess, J., Zumino, B. : Nucl. Phys. B 78, 1-13 (1974) 
2. Salam, A., Strathdee, J.: Nucl. Phys. B 76, 477-482 (1974)

Salam, A., Strathdee, J.: Nucl. Phys. B 80, 499-505 (1974)

Salam, A., Strathdee, J.: Phys. Rev. D11, 1521-1535 (1975)

3. Akulov, V.P., Volkov, D.V., Soroka, V.A.: J.E.T.P. Letters 22, 187-188 (1975)

Wess, J., Zumino, B.: Phys. Lett. 66 B, 361-364 (1977)

4. Chamseddine, A.H., West, P.C.: Nucl. Phys. B129, 39-44 (1977)

MacDowell, S.W., Mansouri, F.: Phys. Rev. Lett. 38, 739-741 (1977)

D'Adda, A., D'Auria, R., Fre' P. Regge, T.: Geometrical formulation of supergravity theories on orthosymplectic supergroup manifolds. Preprint, Torino 1980

5. Kobayashi, S., Nomizu, K.: Foundations of differential geometry, Vols. I, II. New York, London: Interscience Publishers 1963

Daniel, M., Viallet, C.M.: The geometrical setting of gauge theories of the Yang-Mills type. Preprint PAR-LPTHE, 79/03, 1979

6. Steenrod, N.: The topology of fibre bundles, Paragraph 3, $9^{\text {th }}$ edit. Princeton, New Jersey: Princeton University Press 1974, pp. 14-16

7. Milner, J.W.: L'Enseignment Math. 9, 198-203 (1963)

Geroch, R.: J. Math. Phys. 9, 1739-1743 (1968)

8. Atiyah, M.F., Bott, R., Patodi, V.K.: Inventiones Math. 19, 279-330 (1973)

Avis, S.J., Isham, C.J.: To appear in Commun. Math. Phys. (1980)

Hawking, S.W., Pope, C.N.: Phys. Lett. 73 B, $42-44$ (1978)

Petry, H.R.: J. Math. Phys. 20, 231-240 (1979)

Whiston, G. : Gen. Relat. Grav. 6, 463-475 (1975)

9. Deser, S., Zumino, B.: Phys. Lett. 62 B, 335-337 (1976)

10. Fradkin, E.S., Fradkina, T.E.: Phys. Lett. 72 B, 343-348 (1978)

11. Brink, L., Gell-Mann, M., Ramond, P., Scwarz, J.H.: Phys. Lett. 74 B, 336-340 (1978)

12. Jackiw, R.: Phys. Rev. Lett. 41, 1635-1637 (1978)

Communicated by R. Geroch

Received February 20, 1980 